\title{
Factores de riesgo relacionados con la salud sexual en los jóvenes europeos
}

\author{
María Calatrava $^{a}$ \\ Cristina López-del Burgo a,b
}

Jokin de Irala ${ }^{a, b, *}$

anstituto de Cultura y Sociedad, Universidad de Navarra, Pamplona, Navarra, España

${ }^{\mathrm{b}}$ Medicina Preventiva y Salud Pública, Facultad de Medicina, Universidad de Navarra, Pamplona, Navarra, España

*Autor para correspondencia:

Tel: +34948425600 (ext. 806428) Fax: +34948425649

Correo electrónico: jdeirala@unav.es

\section{Publicado en:}

Medicina Clínica

(www.elsevier.es/medicinaclinica)

Mirar el artículo original en la página web de la revista:

http://www.sciencedirect.com/science/article/pii/S0025775311008621

\section{Citar como:}

Calatrava M, López-del Burgo C, de Irala J. Factores de riesgo relacionados con la salud sexual en los jóvenes europeos. Medicina Clínica 2012;138(12):534-540. 


\section{Resumen}

En Europa, seguimos asistiendo a un aumento de la transmisión sexual del virus de la inmunodeficiencia humana (VIH) y otras infecciones de transmisión sexual (ITS). Para priorizar estrategias de salud sexual, resulta importante identificar los factores sexuales de riesgo presentes en los jóvenes europeos. Se realizó una revisión sistemática de artículos científicos y estudios de instituciones oficiales europeas. En total, fueron identificados 21 artículos y 10 estudios. Los datos sugieren un aumento de la iniciación sexual juvenil y del número de parejas sexuales. El 15-20\% de los jóvenes usan de forma inconstante el preservativo. Entre los conocimientos y actitudes de riesgo detectadas encontramos: desconocer otras ITS distintas al VIH, tener una actitud favorable a las relaciones sexuales casuales, creer erróneamente que algunas medidas son eficaces para prevenir el VIH, desconocer los riesgos de tener múltiples parejas sexuales y desconocer la transmisión sexual del VIH. Los datos subrayan la necesidad de mejorar los mensajes transmitidos a los jóvenes.

Palabras clave: infecciones de transmisión sexual, jóvenes, Europa, prácticas sexuales, factores de riesgo 


\section{Introducción}

En los últimos años, asistimos a un incremento mundial de las infecciones de transmisión sexual (ITS), siendo especialmente significativo entre los jóvenes ${ }^{1}$. En 2009 , se produjeron más de 2,2 millones de nuevas infecciones por VIH en personas mayores de 15 años, siendo el $41 \%$ de ellas en jóvenes de 15-24 años ${ }^{2}$.

En Europa, las ITS también constituyen un problema importante para la Salud Pública. Recientemente, el Centro Europeo de Vigilancia Epidemiológica del Sida (EuroHIV) ha comunicado que la tasa de nuevos diagnósticos de infección por VIH en la Unión Europea se ha duplicado entre 1999 y $2009^{3}$.

Los datos españoles de incidencia muestran la misma tendencia ascendente que en el resto de la Unión. Desde el año 2003, se está produciendo un continuado aumento de los diagnósticos de enfermedades de transmisión sexual (ETS) sometidas a vigilancia epidemiológica (gonorrea y sífilis). Destacan, en particular, los datos sobre la incidencia de sífilis. Ésta ha evolucionado de 2,57 por 100.000 habitantes en 1995 a 5,33 por 100.000 en 2009. En cuanto al VIH, su transmisión ha disminuido notablemente respecto a la década de los años 90, donde la transmisión por vía intravenosa era la más frecuente. Actualmente, la vía sexual ha pasado a convertirse en el principal mecanismo de transmisión ${ }^{4}$.

Este empeoramiento general de la situación con respecto a años anteriores parece estar relacionado con un incremento de las prácticas sexuales de riesgo de la población como una iniciación sexual más precoz, el aumento del número de parejas sexuales, el uso inconsistente del preservativo y las relaciones sexuales entre varones ${ }^{5-8}$. La actuación sobre estas prácticas supone un importante reto para la Salud Pública.

A pesar de existir actualmente una evidencia científica consistente sobre la asociación de las prácticas de riesgo arriba mencionadas y el incremento de ITS, sólo una minoría de los jóvenes europeos dispone de una información completa y rigurosa sobre las ITS y su prevención. Por 
ejemplo, en una encuesta nacional realizada en España, menos de la mitad de los jóvenes de 18-19 años $(43,6 \%)$ señalaron que tener pocas parejas era una medida eficaz frente al $\mathrm{VIH}^{9}$. Kouznetsov et al. investigaron el conocimiento que tenía una población de Munich sobre la transmisión sexual del VIH. Los resultados del estudio de Munich concluyen que sólo el 55,5\% de los encuestados conocía que el VIH podría ser transmitido a través de las tres vías de infección (vaginal, anal y oral) ${ }^{10}$. La carencia de conocimientos puede ser mayor en países que están en vías de desarrollo. Por ejemplo, según UNICEF, sólo el 24\% de los jóvenes de 15-24 años en países de bajo y medio desarrollo identifican el uso de preservativos y tener relaciones sexuales mutuamente monógamas con una persona no infectada como medidas de prevención del VIH ${ }^{11}$. Este desconocimiento puede impedir que los jóvenes logren evitar estos riesgos con mayor eficacia.

A fin de priorizar estrategias de prevención de las ITS resulta fundamental identificar las prácticas, conocimientos y actitudes de los jóvenes europeos relativos a la sexualidad que pueden ponerles en riesgo de adquirir una ITS. Para ello, realizamos una revisión bibliográfica de publicaciones y encuestas llevadas a cabo en Europa occidental con información relevante sobre estas cuestiones.

\section{Métodos}

\section{Estrategia de búsqueda}

En primer lugar, se realizó una revisión bibliográfica de artículos científicos a través de la base de datos MEDLINE (PubMed). Como estrategia primaria de búsqueda se utilizaron las siguientes palabras clave "sexual behaviour", "sexual knowledge", "sexual attitudes", "STD”, "STI", “adolescent" y "young adult". Además, se revisaron los artículos relevantes que eran citados en los estudios seleccionados. También se revisaron otros trabajos que citaban a los estudios que habíamos seleccionado a través de la herramienta “Google Académico”. De este modo, pretendíamos asegurar que ningún estudio relevante era omitido.

En un segundo tiempo, se localizaron encuestas sobre sexualidad llevadas a cabo por instituciones sociales o políticas de Europa occidental a través de sus páginas webs oficiales. 
Ambas búsquedas fueron realizadas entre octubre de 2007 y enero de 2010.

Selección de los estudios y encuestas

Los criterios de inclusión de los estudios que iban a formar parte de la revisión fueron los siguientes: (a) desarrollado en un país de Europa occidental, según la división geográfica de la Organización Mundial de la Salud (OMS); (b) con información sobre los conocimientos, actitudes y comportamientos relativos a la sexualidad y la prevención de las ITS; (c) realizado en una muestra de la población general de jóvenes que comprendiera cualquier edad en el rango de 16-20 años; (d) escrito en inglés o castellano; (e) con un tamaño muestral superior a 1000 sujetos, y (e) posteriores a 1999.

Dado que el objetivo es describir los conocimientos, opiniones y prácticas de la "población general de jóvenes" relativos a la sexualidad y la prevención de las ITS, se excluyeron aquellos estudios realizados exclusivamente sobre poblaciones con un mayor riesgo de adquirir una ITS (usuarios de drogas por vía parenteral, población previamente diagnosticada de alguna ITS, varones con relaciones homosexuales, minorías étnicas o personas relacionadas con el comercio del sexo) ${ }^{12}$. También se excluyeron aquellos estudios realizados con muestras de jóvenes que acudían a las clínicas de atención de ITS o a clínicas ginecológicas dado que estos pueden presentar características distintas a las de la "población general de jóvenes"13.

De cada estudio seleccionado se recogió la siguiente información: país, nombre del estudio y año de realización, diseño epidemiológico, tipo de muestreo, rango de edad de la población de estudio, tamaño muestral, factor/es de riesgo de ITS detectado/s (de tipo conceptual, actitudinal o conductual) y su frecuencia en los jóvenes.

En este trabajo nos hemos centrado en los siguientes factores de riesgo independientes para adquirir una ITS: los errores de conocimiento sobre las ITS, las actitudes favorables a las relaciones sexuales casuales, dado el riesgo que éstas conllevan, el inicio precoz de las relaciones sexuales (a menor edad, mayor riesgo), tener múltiples parejas sexuales seriadas o concurrentes, no usar preservativos 
o hacerlo de modo inconsistente (utilizarlo solamente en algunas relaciones sexuales) y tener coitos anales $^{5,6,14}$.

\section{Resultados}

Con la estrategia de búsqueda inicial, se identificaron un total de 1424 artículos, de los cuales, se excluyeron 1180 porque en el título hacían referencia explícita a una población con mayor riesgo de ITS. Tras la lectura de los resúmenes seleccionados, se excluyeron 223 artículos más por no cumplir alguno de los criterios de inclusión. La muestra final de los estudios publicados en revistas contuvo 21 artículos sobre 17 estudios diferentes ${ }^{6-8,15-33}$.

En relación con la búsqueda de encuestas llevadas a cabo por instituciones oficiales y ministerios europeos, se localizaron 10 llevadas a cabo en jóvenes europeos ${ }^{9,34-42}$.

Las características de los estudios y encuestas revisadas se presentan en las tablas 1 y 2, respectivamente. Se presentan nueve estudios aleatorios y cinco realizados en muestras de conveniencia con tamaños muestrales entre 1894 y 100.790 jóvenes (tabla 1). Con respecto a las encuestas, todas son aleatorias excepto una, de conveniencia, con tamaños muestrales entre 1000 y 12364 jóvenes (tabla 2).

A continuación, se describen, por orden de frecuencia, los conocimientos, actitudes y prácticas sexuales de riesgo detectadas en los estudios y encuestas revisadas.

\section{Conocimientos y actitudes de riesgo}

No conocer otras ITS distintas al VIH

Según una encuesta realizada en Irlanda del Norte, sólo uno de cada cuatro jóvenes de 14-25 años conoce otras ITS distintas del $\mathrm{VIH}^{40}$. Cuando se pide a los jóvenes italianos de 14-16 años que identifiquen los mecanismos de transmisión de algunas infecciones, pocos señalan la vía sexual como mecanismo de transmisión de ITS. Por ejemplo, el $42 \%$ desconoce que el Herpes Genital pueda transmitirse sexualmente. Este porcentaje es aún mayor con respecto a la sífilis y la gonorrea $(82,7 \% \text { y } 91,2 \% \text {, respectivamente })^{37}$. 


\section{Tener una actitud favorable a las relaciones sexuales casuales}

A pesar de que las relaciones sexuales casuales son un factor de riesgo para adquirir una ITS, el $57 \%$ de los varones y el $28 \%$ de las mujeres francesas de 18-24 años manifiestan que podrían tener relaciones sexuales con una persona sin que exista amor entre ellos ${ }^{38}$. Más de dos tercios de los jóvenes de Irlanda del Norte de 15-24 años aprueban las relaciones sexuales casuales ${ }^{40}$. Del mismo modo opinan el $73 \%$ de los varones y el $50 \%$ de las mujeres irlandesas de $18-24$ años ${ }^{36}$. El $29 \%$ de los varones y el 36\% de las mujeres suecas de 16-24 años creen que las relaciones sexuales sólo deben tener lugar dentro de una relación estable ${ }^{29}$.

Tabla 1. Características de los estudios seleccionados de revistas científicas (orden alfabético de países)

\begin{tabular}{|c|c|c|c|c|}
\hline $\begin{array}{c}\text { PAÍS } \\
\text { (CIUDAD) }\end{array}$ & NOMBRE, AÑO & MUESTREO & POBLACIÓN & $\begin{array}{c}\text { TAMAÑO } \\
\text { MUESTRAL }\end{array}$ \\
\hline $\begin{array}{l}\text { Bélgica } \\
\text { (Antwerp) }\end{array}$ & Antwerp Study, $1997^{15}$ & Conveniencia & $\begin{array}{l}\text { Mujeres estudiantes } \\
16-18 \text { años }\end{array}$ & 2784 \\
\hline Bélgica & $\begin{array}{l}\text { Flemish Education Assessment Project, } \\
2005^{16}\end{array}$ & $\begin{array}{c}\text { Aleatorio } \\
\text { multietápico }\end{array}$ & $\begin{array}{l}\text { Estudiantes de 14- } \\
16 \text { años }\end{array}$ & 11872 \\
\hline España & Estudio Afrodita, $2005^{6}$ & $\begin{array}{c}\text { Aleatorio } \\
\text { estratificado }\end{array}$ & $\begin{array}{l}\text { Mujeres } \\
18-70 \text { años }\end{array}$ & 6852 \\
\hline Finlandia & $\begin{array}{l}\text { The School Health Promotion } \\
\text { Study, } 2003^{17}\end{array}$ & Conveniencia & $\begin{array}{l}\text { Estudiantes de 14- } \\
16 \text { años }\end{array}$ & 100790 \\
\hline Finlandia & The Health 2000 survey, $2001^{18}$ & $\begin{array}{c}\text { Aleatorio } \\
\text { estratificado }\end{array}$ & $\begin{array}{c}\text { Población general } \\
18-29 \text { años } \\
\end{array}$ & 1894 \\
\hline $\begin{array}{l}\text { Gran } \\
\text { Bretaña }\end{array}$ & SHARE and RIPPLE Studies, $1997^{19}$ & $\begin{array}{c}\text { Aleatorio } \\
\text { estratificado }\end{array}$ & $\begin{array}{l}\text { Jóvenes } \\
\text { 13-16 años }\end{array}$ & 11625 \\
\hline Grecia & EURO-BLCS Greece, $2001^{20}$ & Conveniencia & $\begin{array}{c}\text { Estudiantes } \\
18 \text { años }\end{array}$ & 3373 \\
\hline Italia & $\begin{array}{l}\text { Third italian survey on knowledge, } \\
\text { attitudes and sexual behaviour in relation } \\
\text { to HIV/AIDS risk, } 2002^{21}\end{array}$ & $\begin{array}{c}\text { Aleatorio } \\
\text { estratificado }\end{array}$ & $\begin{array}{l}\text { Población general } \\
\text { 18-49 años }\end{array}$ & 1985 \\
\hline Italia & $\begin{array}{l}\text { La prevenzione mirata al comportamento } \\
\text { sessuale, } 2000^{22}\end{array}$ & $\begin{array}{l}\text { Aleatorio } \\
\text { estratificado }\end{array}$ & $\begin{array}{l}\text { Jóvenes } \\
18-26 \text { años }\end{array}$ & 8533 \\
\hline Italia & Me \& my Health, $2005^{23}$ & Conveniencia & $\begin{array}{l}\text { Estudiantes } \\
14-19 \text { años }\end{array}$ & 2273 \\
\hline $\begin{array}{l}\text { Noruega } \\
\text { (Oslo) }\end{array}$ & The Oslo Youth survey, $1996^{24}$ & Conveniencia & $\begin{array}{l}\text { Estudiantes } \\
16 \text { años }\end{array}$ & 3464 \\
\hline $\begin{array}{l}\text { Reino } \\
\text { Unido } \\
\text { (Londres) } \\
\end{array}$ & Naz Project London ${ }^{25}$ & Conveniencia & $\begin{array}{l}\text { Estudiantes de } 15- \\
\qquad 18 \text { años }\end{array}$ & 2218 \\
\hline $\begin{array}{l}\text { Reino } \\
\text { Unido }\end{array}$ & Natsal, $2000^{7,8,26-28}$ & $\begin{array}{l}\text { Aleatorio } \\
\text { estratificado }\end{array}$ & $\begin{array}{l}\text { Población general } \\
16-44 \text { años }\end{array}$ & 11161 \\
\hline Suecia & KABP survey, $2007^{29}$ & $\begin{array}{l}\text { Aleatorio } \\
\text { simple }\end{array}$ & $\begin{array}{l}\text { Población general } \\
16-44 \text { años }\end{array}$ & 3011 \\
\hline Suecia & $\begin{array}{l}\text { The national cross-sectional SAM 73-90 } \\
\text { questionnaire survey, } 1990^{30,31}\end{array}$ & $\begin{array}{l}\text { Aleatorio } \\
\text { agrupado }\end{array}$ & $\begin{array}{l}\text { Jóvenes } \\
17 \text { años }\end{array}$ & 2757 \\
\hline Suiza & Swiss National Survey, $1996^{32}$ & $\begin{array}{l}\text { Aleatorio } \\
\text { agrupado }\end{array}$ & $\begin{array}{l}\text { Jóvenes } \\
\text { 16-20 años }\end{array}$ & 4283 \\
\hline Varios* & $\begin{array}{l}\text { The Adolescent Sexual Behaviour Survey, } \\
2007^{33}\end{array}$ & $\begin{array}{l}\text { Aleatorio } \\
\text { simple }\end{array}$ & $\begin{array}{l}\text { Jóvenes de } 18-24 \\
\text { años }\end{array}$ & 7657 \\
\hline
\end{tabular}

Nota: todos los estudios son transversales excepto The SHARE \& RIPPLE Studies que es longitudinal

*Francia, Irlanda, Italia, Holanda, Polonia, República Checa y Rusia. 
Tabla 2. Características de las encuestas realizadas por instituciones oficiales europeas

\begin{tabular}{|c|c|c|c|c|}
\hline $\begin{array}{l}\text { PAÍS O } \\
\text { REGIÓN }\end{array}$ & NOMBRE (AÑN) & MUESTREO & POBLACIÓN & $\begin{array}{c}\text { TAMAÑO } \\
\text { MUESTRAL }\end{array}$ \\
\hline España & Juventud en España, $2008^{34}$ & $\begin{array}{l}\text { Aleatorio } \\
\text { estratificado }\end{array}$ & $\begin{array}{l}\text { Jóvenes } \\
15-29 \text { años }\end{array}$ & 5014 \\
\hline España & HSBC España, $2006^{42}$ & $\begin{array}{c}\text { Aleatorio } \\
\text { estratificado }\end{array}$ & $\begin{array}{l}\text { Estudiantes } \\
11-17 \text { años }\end{array}$ & 6727 \\
\hline España & Informe FIPSE, $2006^{9}$ & $\begin{array}{l}\text { Aleatorio } \\
\text { estratificado }\end{array}$ & $\begin{array}{l}\text { Jóvenes } \\
\text { 18-29 años }\end{array}$ & 4135 \\
\hline España & $\begin{array}{l}\text { Sexualidad y anticoncepción en la } \\
\text { juventud española, } 2005^{35}\end{array}$ & Aleatorio & $\begin{array}{c}\text { Jóvenes } \\
15-24 \text { años } \\
\end{array}$ & 2015 \\
\hline Finlandia & The Finish Youth Survey, $2006^{41}$ & $\begin{array}{l}\text { Aleatorio } \\
\text { estratificado }\end{array}$ & $\begin{array}{c}\text { Jóvenes } \\
15-29 \text { años }\end{array}$ & 1901 \\
\hline Francia & CSF Survey, $2007^{38}$ & $\begin{array}{c}\text { Aleatorio } \\
\text { estratificado }\end{array}$ & $\begin{array}{c}\text { Población general } \\
\text { 18-69 años }\end{array}$ & 12364 \\
\hline Italia & $\begin{array}{l}\text { Salute riproduttiva tra gli adolescenti: } \\
\text { conoscenze, attitudini e } \\
\text { comportamenti, } 2000^{37}\end{array}$ & Aleatorio & $\begin{array}{c}\text { Estudiantes } \\
14-16 \text { años }\end{array}$ & 6467 \\
\hline Irlanda & $\begin{array}{l}\text { The Irish study of sexual healh and } \\
\text { relationships, } 2005^{36}\end{array}$ & Aleatorio & $\begin{array}{c}\text { Población general } \\
18-64 \text { años }\end{array}$ & 7441 \\
\hline $\begin{array}{l}\text { Irlanda del } \\
\text { Norte }\end{array}$ & Health and wellbeing survey, $2001^{39}$ & Aleatorio & $\begin{array}{l}\text { Población general } \\
16-44 \text { años }\end{array}$ & 5205 \\
\hline $\begin{array}{l}\text { Irlanda del } \\
\text { Norte }\end{array}$ & Towards better sexual health, $2001^{40}$ & Conveniencia & $\begin{array}{c}\text { Jóvenes } \\
14-25 \text { años }\end{array}$ & 1000 \\
\hline
\end{tabular}

Nota: todos los estudios son transversales

Creer erróneamente que algunas medidas son eficaces para prevenir el VIH

"Preguntar a la pareja por su pasado", "usar espermicidas" o "lavarse tras la relación sexual" son consideradas medidas preventivas eficaces frente al VIH por el $59,3 \%$, el $33 \%$ y el $29,9 \%$ de los jóvenes españoles de 18-19 años, respectivamente ${ }^{9}$.

No conocer los riesgos de tener múltiples parejas sexuales

"Tener pocas parejas" no es considerada una medida eficaz por el 56,4\% de los jóvenes españoles de 18-19 años ${ }^{9}$.

No conocer que el VIH se transmite por vía sexual

Según diversas encuestas, el 3,5\% de los jóvenes españoles de 18-19 años y el 1,6\% de jóvenes italianos de 14-16 años, desconocen que el VIH se transmite por la vía sexual ${ }^{9,37}$.

Prácticas de riesgo

En la tabla 3 se resumen las prácticas sexuales de riesgo más frecuentes entre los jóvenes europeos. 
Tabla 3. Porcentaje de algunas prácticas sexuales de riesgo en jóvenes europeos

\begin{tabular}{|c|c|c|c|c|c|c|c|c|}
\hline \multirow[b]{3}{*}{$\begin{array}{c}\text { PAÍS } \\
\text { Encuesta (título abreviado), año }\end{array}$} & \multirow{3}{*}{$\begin{array}{c}\text { Rango de } \\
\text { edad } \\
\text { (años) } \\
\end{array}$} & \multicolumn{7}{|c|}{ PRÁCTICAS SEXUALES } \\
\hline & & \multicolumn{3}{|c|}{$\begin{array}{c}\text { Han tenido ya } \\
\text { relaciones sexuales } \\
\text { completas }(\%)\end{array}$} & \multicolumn{3}{|c|}{$\begin{array}{c}\text { Han tenido } \\
\text { múltiples parejas } \\
(2 \text { o más })(\%)\end{array}$} & \multirow{2}{*}{$\begin{array}{c}\text { Han hecho un uso } \\
\text { inconsistente del } \\
\text { preservativo* }(\%)\end{array}$} \\
\hline & & 0 & q & Total & $\hat{0}$ & q & Total & \\
\hline \multicolumn{9}{|l|}{ BÉLGICA } \\
\hline Antwerp Study, $1997^{15}$ & $15-23$ & ND & 52 & ND & ND & $\begin{array}{l}24,7^{\mathrm{a}} \\
54,3^{\mathrm{b}}\end{array}$ & ND & $79,4^{\mathrm{b}} ; 20^{\mathrm{c}} ; 63,5^{\mathrm{d}}$ \\
\hline Flemish Education Assessment project ${ }^{16}$ & $14-16$ & ND & ND & 28,6 & ND & ND & ND & $69,9^{\mathbf{b}}$ \\
\hline \multicolumn{9}{|l|}{ ESPAÑA } \\
\hline Juventud en España, $2008^{34}$ & $\begin{array}{l}15-17 \\
18-20\end{array}$ & $\begin{array}{l}42,4 \\
85,9\end{array}$ & $\begin{array}{l}39,9 \\
74,1\end{array}$ & $\begin{array}{c}42,4 \\
80\end{array}$ & $\begin{array}{l}40,5^{\mathrm{a}} \\
43,3^{\mathrm{a}}\end{array}$ & $\begin{array}{l}12,8^{\mathrm{a}} \\
16,4^{\mathrm{a}}\end{array}$ & $\begin{array}{l}\text { ND } \\
\text { ND }\end{array}$ & $\begin{array}{l}25,8^{\mathrm{a}} \\
34,3^{\mathrm{a}}\end{array}$ \\
\hline HSBC España, 2006 $6^{42}$ & $\begin{array}{l}15-16 \\
17-18 \\
\end{array}$ & $\begin{array}{l}27,8 \\
43,4 \\
\end{array}$ & $\begin{array}{l}25,4 \\
45,9\end{array}$ & $\begin{array}{l}\text { ND } \\
\text { ND }\end{array}$ & $\begin{array}{c}54^{\mathrm{b}} \\
50,2^{\mathrm{b}}\end{array}$ & $\begin{array}{l}28,6^{\mathrm{b}} \\
33,3^{\mathrm{b}}\end{array}$ & $\begin{array}{l}\text { ND } \\
\text { ND }\end{array}$ & ND \\
\hline Informe FIPSE, $2006^{9}$ & 18-19 & 75,6 & 69,1 & ND & $\begin{array}{l}49,2^{\mathrm{b}} \\
14,9^{\mathrm{ae}}\end{array}$ & $\begin{array}{l}27,7^{\mathrm{b}} \\
11,1^{\text {ae }}\end{array}$ & ND & $12,5^{\mathrm{c}} ; 20,2^{\mathrm{d}}$ \\
\hline Sexualidad y anticoncepción, $2005^{35}$ & $15-19$ & 55,8 & 52,8 & ND & $\mathrm{ND}$ & ND & ND & $26,5^{\mathrm{b}}$ \\
\hline Estudio Afrodita, $2005^{6}$ & $18-25$ & $\mathrm{NE}$ & 75,9 & ND & ND & $42,2^{\mathrm{b}}$ & ND & ND \\
\hline \multicolumn{9}{|l|}{ FINLANDIA } \\
\hline The School Health Promotion Study, 2003 & $\begin{array}{l}14-15 \\
15-16\end{array}$ & $\begin{array}{l}16 \\
27\end{array}$ & $\begin{array}{l}18 \\
34\end{array}$ & $\begin{array}{l}\text { ND } \\
\text { ND }\end{array}$ & $\begin{array}{l}51^{\mathrm{b}} \\
54^{\mathrm{b}}\end{array}$ & $\begin{array}{l}48^{\mathrm{b}} \\
54^{\mathrm{b}}\end{array}$ & $\begin{array}{l}\text { ND } \\
\text { ND }\end{array}$ & ND \\
\hline The Health 2000 survey $^{18}$ & $\begin{array}{l}18-19 \\
20-24\end{array}$ & $\begin{array}{l}73 \\
88\end{array}$ & $\begin{array}{l}85 \\
89\end{array}$ & $\begin{array}{c}79 \\
88,5\end{array}$ & $\begin{array}{l}33^{\mathrm{b}} \\
29^{\mathrm{b}}\end{array}$ & $\begin{array}{l}21^{\mathrm{b}} \\
20^{\mathrm{b}}\end{array}$ & $\begin{array}{l}\text { ND } \\
\text { ND }\end{array}$ & $\begin{array}{l}40^{\mathrm{d}} \\
55^{\mathrm{d}}\end{array}$ \\
\hline \multicolumn{9}{|l|}{ FRANCIA } \\
\hline $\begin{array}{l}\text { The Adolescent Sexual Behaviour } \\
\text { Survey, } 2007^{33}\end{array}$ & $18-24$ & ND & ND & ND & ND & ND & ND & $11,8^{\mathbf{c}}(\mathrm{V}) ; 17,7^{\mathbf{c}}(\mathrm{M})$ \\
\hline ASCF Survey, $2007^{38}$ & $\begin{array}{l}18-19 \\
20-24 \\
18-24 \\
\end{array}$ & $\begin{array}{l}\mathrm{ND} \\
\mathrm{ND} \\
\mathrm{ND} \\
\end{array}$ & $\begin{array}{l}\text { ND } \\
\text { ND } \\
\text { ND } \\
\end{array}$ & $\begin{array}{l}\text { ND } \\
\text { ND } \\
\text { ND } \\
\end{array}$ & $\begin{array}{c}27,5^{\mathrm{a}} \\
31,7^{\mathrm{a}} \\
\mathrm{ND} \\
\end{array}$ & $\begin{array}{c}22,3^{\mathrm{a}} \\
19^{\mathrm{a}} \\
\mathrm{ND} \\
\end{array}$ & $\begin{array}{l}\text { ND } \\
\text { ND } \\
\text { ND } \\
\end{array}$ & $\begin{array}{c}\mathrm{ND} \\
\mathrm{ND} \\
11,5^{\mathrm{c}} \\
\end{array}$ \\
\hline \multicolumn{9}{|l|}{ GRECIA } \\
\hline EURO-BLCS Greece, $2001^{20}$ & 18 & ND & ND & 38 & ND & ND & ND & ND \\
\hline \multicolumn{9}{|l|}{ HOLANDA } \\
\hline $\begin{array}{l}\text { The Adolescent Sexual Behaviour } \\
\text { Survey, } 2007^{33}\end{array}$ & $18-24$ & ND & ND & ND & ND & ND & ND & $24,4^{\mathbf{c}}(\mathrm{V}) ; 17,2^{\mathbf{c}}(\mathrm{M})$ \\
\hline \multicolumn{9}{|l|}{ IRLANDA } \\
\hline $\begin{array}{l}\text { The Adolescent Sexual Behaviour } \\
\text { Survey, } 2007^{33}\end{array}$ & $18-24$ & ND & ND & ND & ND & ND & ND & $25,4^{\mathbf{c}}(\mathrm{V}) ; 16,2^{\mathbf{c}}(\mathrm{M})$ \\
\hline The Irish study, $2005^{36}$ & $18-24$ & 84,3 & 81,8 & 83 & $66^{\mathrm{b}}$ & $50^{\mathrm{b}}$ & ND & $46^{\mathrm{a}} ; 28^{\mathrm{d}}$ \\
\hline
\end{tabular}


Tabla 3. Porcentaje de algunas prácticas sexuales de riesgo en jóvenes europeos (continuación)

\begin{tabular}{|c|c|c|c|c|c|c|c|c|}
\hline \multirow[b]{3}{*}{$\begin{array}{c}\text { PAÍS } \\
\text { Encuesta (título abreviado), año }\end{array}$} & \multirow[b]{3}{*}{$\begin{array}{l}\text { Rango de } \\
\text { edad } \\
\text { (años) }\end{array}$} & \multicolumn{7}{|c|}{ PRÁCTICAS SEXUALES } \\
\hline & & \multicolumn{3}{|c|}{$\begin{array}{c}\text { Han tenido ya } \\
\text { relaciones sexuales } \\
\text { completas }(\%)\end{array}$} & \multicolumn{3}{|c|}{$\begin{array}{c}\text { Han tenido } \\
\text { múltiples parejas } \\
(2 \text { o más })(\%)\end{array}$} & \multirow{2}{*}{$\begin{array}{l}\text { Han hecho un uso } \\
\text { inconsistente del } \\
\text { preservativo* }(\%)\end{array}$} \\
\hline & & $\lambda$ & q & Total & $\widehat{\delta}$ & 우 & Total & \\
\hline \multicolumn{9}{|l|}{ ITALIA } \\
\hline $\begin{array}{l}\text { The Adolescent Sexual Behaviour } \\
\text { Survey, } 2007^{33}\end{array}$ & $18-24$ & ND & ND & ND & ND & ND & ND & $28,3^{\mathbf{c}}(\mathrm{V}) ; 30,1^{\mathbf{c}}(\mathrm{M})$ \\
\hline Me \& my Health, $2005^{23}$ & $14-19$ & ND & ND & 30 & $\mathrm{ND}$ & ND & ND & ND \\
\hline 3 rd italian survey, $2002^{21}$ & $18-24$ & ND & ND & 73,7 & ND & ND & $\mathrm{ND}$ & ND \\
\hline $\begin{array}{l}\text { La prevenzione mirata al comportamento } \\
\text { sessuale, } 2000^{22}\end{array}$ & $18-26$ & 70 & 64 & 67 & ND & ND & ND & $7^{\mathrm{d}}$ \\
\hline $\begin{array}{l}\text { Salute reproductiva tra gli adolescenti, } \\
2000^{37}\end{array}$ & $14-16$ & 23,8 & 12,1 & 18 & ND & ND & ND & $45^{\mathrm{b}}$ \\
\hline \multicolumn{9}{|l|}{ NORUEGA } \\
\hline The Oslo Youth survey, $1996^{24}$ & 16 & 49,8 & 50,2 & 50 & $\mathrm{ND}$ & ND & ND & ND \\
\hline \multicolumn{9}{|l|}{ POLONIA } \\
\hline $\begin{array}{l}\text { The Adolescent Sexual Behaviour } \\
\text { Survey, } 2007^{33}\end{array}$ & $18-24$ & ND & ND & ND & ND & ND & ND & $22,8^{\mathbf{c}}(\mathrm{V}) ; 24,6^{\mathbf{c}}(\mathrm{M})$ \\
\hline \multicolumn{9}{|l|}{ REINO UNIDO } \\
\hline Naz Project London ${ }^{25}$ & $15-18$ & 37,2 & 23,9 & 30,5 & $\mathrm{ND}$ & ND & ND & ND \\
\hline Health and wellbeing survey, $2001^{39}$ & $16-24$ & 70 & 68 & 69 & ND & ND & ND & ND \\
\hline Towards better sexual Health, $2001^{40}$ & $14-25$ & ND & ND & 53,3 & ND & ND & ND & ND \\
\hline The national survey, $2000^{7,8,26-28}$ & $\begin{array}{l}16-19 \\
16-24\end{array}$ & $\begin{array}{l}\text { ND } \\
\text { ND }\end{array}$ & $\begin{array}{l}\text { ND } \\
\text { ND }\end{array}$ & $\begin{array}{l}\mathrm{ND} \\
\mathrm{ND}\end{array}$ & $\begin{array}{l}\mathrm{ND} \\
65,5^{\mathrm{b}}\end{array}$ & $\begin{array}{l}20,8^{\text {ae }} \\
64,3^{\text {b }}\end{array}$ & $\begin{array}{l}\mathrm{ND} \\
\mathrm{ND}\end{array}$ & $\begin{array}{c}19,7^{\mathbf{c}} \\
\mathrm{ND}\end{array}$ \\
\hline SHARE and RIPPLE Studies, $1997^{19}$ & $13-16$ & 38 & 46 & 42 & $62^{\mathrm{b}}$ & $59^{\mathrm{b}}$ & ND & $29^{\mathrm{c}} ; 38^{\mathrm{d}}$ \\
\hline \multicolumn{9}{|l|}{ REPÚBLICA CHECA } \\
\hline $\begin{array}{l}\text { The Adolescent Sexual Behaviour } \\
\text { Survey, } 2007^{33}\end{array}$ & $18-24$ & ND & ND & ND & ND & ND & ND & $41,9^{\mathbf{c}}(\mathrm{V}) ; 31,4^{\mathbf{c}}(\mathrm{M})$ \\
\hline \multicolumn{9}{|l|}{ SUECIA } \\
\hline KABP survey, $2007^{59}$ & $16-24$ & ND & ND & ND & $21^{\mathrm{b}}$ & $20^{\mathrm{b}}$ & ND & ND \\
\hline Nacional cross-sectional survey, $1990^{30,31}$ & 17 & 54,2 & 64 & 58,9 & ND & ND & ND & ND \\
\hline \multicolumn{9}{|l|}{ SUIZA } \\
\hline Encuesta nacional, $1996^{32}$ & $16-20$ & 50,4 & 52,6 & 51,5 & $41^{\mathrm{b}}$ & $53^{\mathrm{b}}$ & ND & $25^{\mathrm{c}}$ \\
\hline
\end{tabular}

\section{ND: Dato no disponible}

V: Varones; M: Mujeres

*Uso inconsistente es definido como no usar un preservativo en cualquier tipo de acto sexual -vaginal, anal y oral- ${ }^{60}$ (en esta tabla el uso inconsistente del preservativo se refiere exclusivamente a relaciones sexuales vaginales).

${ }^{a}$ En el último año

${ }^{\text {b }}$ A lo largo de la vida

${ }^{\mathrm{c}}$ En la primera relación sexual

${ }^{\mathrm{d}}$ En la última relación sexual

${ }^{\mathrm{e}}$ Parejas concurrentes

Inicio precoz de las relaciones sexuales

La edad de inicio de las relaciones sexuales es un factor de riesgo para contraer una ITS. Cuanto menor es la edad, mayor es el riesgo de contagio ${ }^{5,6}$. 
El porcentaje de jóvenes que tuvieron relaciones sexuales completas con 16 años o menos oscila entre el $16 \%$ (varones) y el 18\% (mujeres) de los jóvenes finlandeses de 14-15 años y el 49,8\% (varones) y el 52,2\% (mujeres) de los jóvenes noruegos de 16 años ${ }^{24,43}$. La proporción de varones que se ha iniciado sexualmente es mayor con respecto a la de mujeres en España, Irlanda e Italia $^{9,21,34-41}$. En Finlandia, Noruega, Suecia y Suiza la proporción de mujeres es mayor a la de $\operatorname{varones}^{17,18,30-32}$.

\section{Tener múltiples parejas sexuales seriadas o concurrentes}

El número de parejas sexuales es un factor de riesgo para contraer una ITS. Cuanto mayor es el número de parejas, mayor es el riesgo de contagio ${ }^{6,7}$.

Una alta proporción de jóvenes europeos refiere haber tenido múltiples parejas sexuales (dos o más) a lo largo de la vida. Las diferencias entre ambos sexos son notables. Los varones suelen tener más parejas sexuales que las mujeres. Por ejemplo, en España, casi la mitad de los varones y sólo una cuarta parte de las mujeres entre 15-20 años ha tenido más de una pareja sexual en su vida ${ }^{34}$. Únicamente en Suiza, la proporción de mujeres de 16-20 años supera a la de varones y en Finlandia, la proporción de mujeres de 15-16 años iguala a la de varones ${ }^{3217}$. Del mismo modo, el número de parejas sexuales en el último año también es mayor en los varones, en todos los estudios que lo preguntan.

Los porcentajes que se dan a continuación se refieren a jóvenes que han tenido ya relaciones sexuales completas.

\section{Tener relaciones sexuales sin preservativo}

En general, un alto porcentaje de los jóvenes refieren utilizar el preservativo de manera inconsistente. El porcentaje de jóvenes que no utilizaron un preservativo en su primera relación sexual varía del 11,5\% en jóvenes franceses de 18-24 años al 29\% en jóvenes británicos de 13-16 años $^{19,38}$. El uso que los jóvenes hacen del preservativo en la última relación sexual es menor que en la primera relación. Así por ejemplo, en España, el 12,5\% de los jóvenes de 18-19 años no usó un 
preservativo en su primera relación sexual mientras un 20,2\% no lo usó la última vez que tuvo relaciones ${ }^{9}$.

\section{Tener sexo comercial}

El 1,3\% de los varones franceses de 18-19 años y el 4\% de los varones irlandeses de 18-24 años refirieron haber tenido sexo comercial $^{36}$.Este porcentaje asciende al $6,1 \%$ entre los jóvenes franceses de 20-24 años y al 10,4\% entre los varones españoles de 18-19 años ${ }^{9,38}$. Asimismo, el 3,4\% de los varones ingleses de 16-24 años refirió haber acudido al sexo comercial en los últimos 5 $\operatorname{años}^{26}$.

\section{Tener coitos anales}

En España, el 2,6\% de los varones de 15-17 años refirió haber tenido coitos anales con personas de su mismo sexo $^{34}$. Esta misma práctica fue también referida por el 4,3\% de los varones españoles de 18-19 años ${ }^{9}$. En Francia, este porcentaje es del 3,8\% entre los varones de 18-24 años ${ }^{38}$. El porcentaje más bajo se ha encontrado en Irlanda, donde el 2,6\% de los varones de 18-24 años refieren esta práctica ${ }^{36}$.

Un único estudio aporta información sobre esta práctica sexual en personas heterosexuales. Entre los jóvenes londinenses de 15-18 años que habían tenido ya relaciones sexuales, aproximadamente el $13 \%$ refirió esta práctica ${ }^{25}$.

\section{Discusión}

Los adolescentes europeos continúan siendo una población vulnerable frente a las ITS. A pesar de los grandes esfuerzos que se están llevando a cabo para mejorar la salud sexual y reproductiva de la población europea, algunos países de Europa occidental como Reino Unido, Suecia, Noruega o Dinamarca siguen teniendo elevadas tasas de ITS entre los jóvenes ${ }^{44}$. Este hecho parece estar relacionado con la presencia de algunas variables de riesgo, tal y como se recoge en este trabajo.

En Europa, parece un hecho evidente el aumento de las prácticas sexuales entre los jóvenes. Según las investigaciones revisadas, una mayoría de jóvenes menores de 25 años ha tenido ya relaciones sexuales. Antes, los varones europeos se iniciaban sexualmente antes que las mujeres europeas. En 
los últimos años, las medias de edad de iniciación sexual de varones y mujeres se han aproximado en algunos países, como por ejemplo, en España ${ }^{34}$.E incluso, algunas encuestas realizadas en Suecia, Noruega, Finlandia, Gran Bretaña y Suiza indican que la proporción de mujeres que ha tenido relaciones sexuales supera a la de varones ${ }^{18,19,30-32}$.

En general, entre los jóvenes iniciados sexualmente, el porcentaje que ha tenido 2 o más parejas sexuales es mayor que hace una década. En la actualidad, los varones siguen teniendo más parejas sexuales que las mujeres. Sin embargo, la proporción de mujeres jóvenes con múltiples parejas sexuales ha aumentado ${ }^{6}$. La proporción de varones que han tenido múltiples parejas sexuales $(2$ o más) se aproxima al cincuenta por ciento en algunos países como España y Finlandia. La proporción más alta de mujeres se ha encontrado en Finlandia donde el $54 \%$ de las mujeres finlandesas de 15-16 años ha tenido relaciones sexuales con varias parejas.

Estos datos sugieren una mayor igualdad de los patrones de conducta sexual entre varones y mujeres. Estos nuevos patrones se caracterizan en la actualidad por una mayor precocidad en la iniciación sexual juvenil y un mayor número de parejas sexuales durante la adolescencia.

Además, junto a estos riesgos encontramos que, en ocasiones, los jóvenes mantienen relaciones sexuales en las que no se utiliza un preservativo. En general, se aprecia una inconsistencia en torno al $15-20 \%$. Estos jóvenes podrían no estar reduciendo el riesgo de adquirir una ITS dado que la evidencia muestra que el preservativo es una medida eficaz para reducir el contagio (sin eliminarlo del todo) cuando se utiliza correctamente y de modo consistente ${ }^{45}$.

Algunos autores han señalado que estas prácticas de riesgo son la principal causa del aumento de las ITS entre los jóvenes. Según Fenton y Hughes, el descenso de la edad de la primera relación sexual, el aumento del número de parejas sexuales a lo largo de la vida (seriadas o concurrentes) y las prácticas sexuales anales entre varones son factores que han contribuido especialmente al aumento de la incidencia de ITS en el Reino Unido ${ }^{14}$.

Obviamente, estas prácticas no son independientes unas de otras. Estudios previos han relacionado el inicio precoz de la actividad sexual con otras prácticas sexuales de riesgo. Los adolescentes que 
se inician a edades muy tempranas refieren con mayor frecuencia tener una ITS, un mayor número de parejas sexuales y un menor uso del preservativo ${ }^{6,46}$. Por tanto, parece lógico pensar que si se consigue un retraso de la edad de iniciación sexual, podría reducirse la frecuencia de estas otras prácticas de riesgo. En este sentido, se ha recomendado ya que es preferible usar la proporción de jóvenes que son sexualmente activos a distintas edades en lugar de la media de edad de inicio de las relaciones sexuales ${ }^{47}$. El parámetro de la media puede proporcionar a los jóvenes una falsa sensación de que la mayoría de los jóvenes ya han tenido relaciones sexuales. Y, como es sabido, las creencias y estereotipos sociales influyen en el comportamiento sexual de los jóvenes ${ }^{48}$. Por este motivo, las encuestas de salud sexual y los medios de comunicación que las divulguen deberían considerar este aspecto a la hora de dar a conocer los resultados de un estudio.

Por otra parte, se han detectado algunos conocimientos erróneos y actitudes arriesgadas. Estos son, por orden de mayor a menor frecuencia: no conocer otras ITS distintas al VIH, tener una actitud favorable a las relaciones sexuales casuales, creer erróneamente que algunas medidas son eficaces para prevenir el VIH ("preguntar a la pareja por su pasado", "usar espermicidas" y "lavarse tras la relación sexual"), no conocer los riesgos de tener múltiples parejas sexuales y no conocer que el VIH se transmite por vía sexual.

La alta prevalencia de errores entre los jóvenes llama especialmente la atención porque estos tienen muchos medios de información a su alcance. Por tanto, no se debe asumir que un mayor acceso a la información sobre sexualidad se traduce necesariamente en formación sobre prevención de ITS.

Puede observarse, por ejemplo, cómo algunas campañas europeas de Salud Sexual están obviando información sobre otras medidas eficaces frente a las ITS distintas al preservativo, como el retraso del inicio de las relaciones sexuales y la reducción del número de parejas sexuales ${ }^{49,50}$. Sin embargo, promocionar también estas medidas de evitación del riesgo puede resultar en una estrategia más completa y de mayor alcance ${ }^{51}$. Algunos estudios epidemiológicos muestran la importancia del retraso en el inicio de las relaciones sexuales y de la monogamia mutua en la reducción de muertes atribuibles al comportamiento sexual ${ }^{52}$. Según el último estudio de Onusida, la incidencia de VIH ha 
disminuido en el año 2009. Éste es un dato significativo que podría estar relacionado con el descenso global del número de parejas sexuales que es indicado en este mismo informe².

La situación de los jóvenes descrita en este artículo puede sugerir también que, a veces, la información que les llega puede ser parcial, sesgada o contiene errores. Se observa, por ejemplo, que muchos de los contenidos televisivos a los que acceden los jóvenes españoles, incluso libros de texto escolares, no informan sobre las consecuencias negativas derivadas de la actividad sexual juvenil (embarazos, ITS,...) $)^{53,54}$. Otros estudios internacionales advierten de que los medios pueden presentar una imagen negativa de los jóvenes que no se han iniciado sexualmente y transmitir a todos ellos la idea de que la actividad sexual es común, casual y está libre de consecuencias ${ }^{55}$. Algunos autores han destacado que los medios tienden a ofrecer escasa información sobre las posibles consecuencias negativas de la actividad sexual juvenil, a pesar de que frecuentemente representan escenas de amor y sexualidad ${ }^{56}$. Incluso, algunos estudios previos señalan que percibir que los medios de comunicación apoyan el comportamiento sexual adolescente aumenta la frecuencia de iniciación sexual precoz ${ }^{57}$.

Vistos los factores de riesgo identificados y su alta frecuencia entre los jóvenes, no puede concluirse que la información esté completamente informada sobre las medidas más eficaces de prevención de las ITS. Esta situación pone de relieve la necesidad de revisar los mensajes y la información que se les transmiten a través de los medios de comunicación y las campañas de prevención de ITS.

Esta revisión presenta varias limitaciones. Debido a la falta de preguntas y encuestas estandarizadas, ha resultado muy complejo realizar comentarios sobre la tendencia de ciertas prácticas sexuales, como por ejemplo, el uso del preservativo en los jóvenes. La mayoría de los datos sobre este tema provienen de encuestas que investigan amplios grupos de gente en edad reproductiva (de 15-44 años o 15-49 años). Además, estos estudios no suelen dar información sobre el uso del preservativo exclusivamente y prefieren dar los datos sobre uso de métodos anticonceptivos (englobando a éste) ${ }^{58}$. 
La comparación entre países ha de realizarse con cautela ya que las encuestas analizadas no han sido realizadas en jóvenes de la misma franja de edad ni han tenido lugar en el mismo momento del tiempo. También debemos ser prudentes a la hora de generalizar estos resultados a toda la población de jóvenes debido a que algunos estudios se llevaron a cabo en muestras de conveniencia, que pueden ser poco representativas de la población general y no permiten generalizar sus conclusiones a la población de referencia ${ }^{15,20,23,24,40}$. Sin embargo, el objetivo de esta revisión no es dar recomendaciones precisas sobre Salud Sexual sino describir, de un modo general, los conocimientos, actitudes y prácticas sexuales de los jóvenes europeos. Los datos recogidos en este trabajo ofrecen una visión global sobre el estado actual de la Salud Sexual en Europa. Y esta aproximación a la realidad juvenil puede servir de fuente de información sobre aspectos a tener en cuenta en los futuros programas de salud sexual y reproductiva en nuestro continente.

Por último, habría que tener en cuenta que no todas las encuestas recogen información sobre todas las variables de riesgo mostradas por lo que se puede estar subestimando la frecuencia de un problema. Por tanto, cabe pensar que los riesgos a los que están expuestos los jóvenes podrían ser aún mayores.

A pesar de las limitaciones descritas, nuestra revisión cuenta con importantes fortalezas. Desde nuestro conocimiento, se trata de la primera revisión sistemática de estudios sobre los conocimientos, opiniones y prácticas sobre sexualidad y prevención de ITS en jóvenes de Europa occidental. Por otra parte, los estudios seleccionados tienen un buen diseño y se han realizado en muestras de la población general de gran tamaño.

En conclusión, los resultados hallados subrayan la necesidad de incidir más en dar una información completa sobre los principales factores de riesgo y de prevención de las ITS. En concreto, una mejora de la salud sexual juvenil se asociará más con el aumento de la edad de inicio de las relaciones sexuales y la reducción del el número de parejas sexuales entre los jóvenes. Las campañas de Salud Pública pueden ser más concretas y claras a la hora de transmitir estos mensajes de evitación del riesgo. 


\section{Financiación}

La Asociación de Amigos de la Universidad de Navarra concedió una beca a la primera autora de este artículo.

El Gobierno de Navarra y el Instituto de Ciencias para la Familia de la Universidad de Navarra destinaron financiación para la realización de este trabajo.

\section{Conflictos de intereses}

Los autores declaran no tener ningún conflicto de intereses.

\section{Agradecimientos}

A la Asociación de Amigos de la Universidad de Navarra, al Gobierno de Navarra y al Instituto de Ciencias para la Familia de la Universidad de Navarra por la financiación otorgada. 


\section{Bibliografía}

1. Monasch R and Mahy M. Young people: the centre of the HIV epidemic. World Health Organ.Tech.Rep. 2006:15.

2. UNAIDS. 2010 Report on the global AIDS epidemic [revista electrónica] [consultado December 15-2010]: Disponible en: http://www.unaids.org/globalreport/Global_report.htm.

3. European Centre for Disease Prevention and Control. ECDC HIV and AIDS in the European Union, 20092010.

4. Instituto de Salud Carlos III. Centro Nacional de Epidemiología. Vigilancia Epidemiológica de las Infecciones de Transmisión Sexual: 1995-2007 2009.

5. Louie KS, de Sanjose S, Diaz M, Castellsague X, Herrero R, Meijer CJ. et al. Early age at first sexual intercourse and early pregnancy are risk factors for cervical cancer in developing countries . Br J Cancer 2009;7:1191-7.

6. de Sanjose S, Cortes X and Mendez Cea. Age at sexual initiation and number of sexual partners in the female Spanish population Results from the AFRODITA survey. European journal of obstetrics, gynecology and reproductive biology 2008;2:234.

7. Johnson AM, Mercer CH, Erens B, Copas AJ, McManus S, Wellings K. et al. Sexual behaviour in Britain: partnerships, practices, and HIV risk behaviours. Lancet 2001;9296:1835-42.

8. Wellings F, Nanchahal K, Macdowall W, McManus S, Erens B, Mercer CH. et al. Sexual behaviour in Britain: early heterosexual experience. Lancet 2001;9296:1843-50.

9. Fundación para la investigación y prevención del sida en España. Jóvenes, relaciones sexuales y riesgo de infeccioón por VIH. Encuesta de salud y hábitos sexuales. España, 2003. Informe FIPSE 2006.

10. Kouznetsov L, Zippel S and Kuznetsov A. What is the accurate knowledge of the German population regarding sexual HIV transmission?. International Journal of Public Health 2009;3:1935.

11. UNICEF. The State Of The World's Children 2006. Excluded and invisible. New York: UNICEF, 2005.

12. Hughes G, Brady AR, Catchpole MA, Fenton KA, Rogers PA, Kinghorn GR. et al. Characteristics of those who repeatedly acquire sexually transmitted infections: a retrospective cohort study of attendees at three urban sexually transmitted disease clinics in England. Sex.Transm.Dis. 2001;7:379-86.

13. Manhart LE, Aral SO and Holmes KKea. Influence of study population on the identification of risk factors for sexually transmitted diseases using a case-control design: The example of gonorrhea. American journal of epidemiology 2004;4:393.

14. Fenton KA and Hughes G. Sexual behaviour in Britain: why sexually.transmitted infections are common. Clinical medicine 2003;3:199. 
15. Vuylsteke B, Vandenbruaene M, Vandenbalcke P, Van Dyck E and Laga M. Chlamydia trachomatis prevalence and sexual behaviour among female adolescents in Belgium. Sex.Transm.Infect.; Sex.Transm.Infect. 1999;3:152-5.

16. Berten $\mathrm{H}$ and Van Rossem R. Doing worse but knowing better: An exploration of the relationship between HIV/AIDS knowledge and sexual behavior among adolescents in Flemish secondary schools. J.Adolesc. 2009;5:1303-19.

17. Lavikainen HM, Lintonen $\mathrm{T}$ and Kosunen E. Sexual behavior and drinking style among teenagers: a population-based study in Finland. Health Promotion International 2009;2:108-19.

18. Nikula M, Koponen P, Haavio-Mannila E and Hemminki E. Sexual health among young adults in Finland: assessing risk and protective behaviour through a general health survey. Scandinavian journal of public health 2007;3:298.

19. Wight D, Parkes A, Strange V, Allen E, Bonell C and Henderson M. The Quality of Young People's Heterosexual Relationships: A Longitudinal Analysis of Characteristics Shaping Subjective Experience. Perspect Sex Reprod Health 2008;4:226-37.

20. Kapi A, Veltsista A, Kavadias G, Lekea V and Bakoula C. Social determinants of self-reported emotional and behavioral problems in Greek adolescents. Social psychiatry and psychiatric epidemiology 2007;7:594.

21. Signorelli C, Pasquarella C, Limina RM, Colzani E, Fanti M, Cielo A. et al. Third Italian national survey on knowledge, attitudes, and sexual behaviour in relation to HIV/AIDS risk and the role of health education campaigns. Eur.J.Public Health 2006;5:498-504.

22. Signorelli C, Renzi C and Zantedeschi EB, A. La prevenizione mirata al comportamento sessuale. Ann.Ist.Super.Sanitá 2000;4:441-3.

23. Ciairano S, Bonino S, Kliewer W, Miceli R and Jackson S. Dating, sexual activity, and wellbeing in Italian adolescents. J.Clin.Child.Adolesc.Psychol. 2006;2:275-82.

24. Valle A, Torgersen L, Røysamb E, Klepp K and Thelle DS. Social class, gender and psychosocial predictors for early sexual debut among 16 year olds in Oslo. The European journal of public health 2005;2:185.

25. Coleman L and Testa A. Experience of sexual intercourse and reported risk behaviour among an ethnically diverse sample of young people. Sex.Health. 2006;3:169-77.

26. Ward H, Mercer C, Wellings D, Fenton K, Erens B, Copas A. et al. Who pays for sex? An analysis of the increasing prevalence of female commercial sex contacts among men in Britain. Sex.Transm.Infect. 2005:467-71.

27. Macdowall W, Wellings K, Mercer CH, Nanchahal K, Copas AJ, McManus S. et al. Learning about sex: Results from Natsal 2000. Health Education \& Behavior 2006;6:802-11.

28. Cassell JA, Mercer CH, Imrie J, Copas AJ and Johnson AM. Who uses condoms with whom? Evidence from national probability sample surveys. Sex.Transm.Infect. 2006;6:467-73.

29. Herlitz $\mathrm{C}$ and Forsberg M. Sexual behaviour and risk assessment in different age cohorts in the general population of Sweden (1989-2007). Scandinavian Journal of Public Health 2010:32-9. 
30. Edgardh K. Sexual behaviour and early coitarche in a national sample of 17-year-old Swedish boys. Acta pædiatrica 2002;9:985.

31. Edgardh K. Sexual behaviour and early coitarche in a national sample of 17 year old Swedish girls. Sexually transmitted infections 2000;2:98.

32. Narring F, Wydler $\mathrm{H}$ and Michaud PA. First sexual intercourse and contraception: a crosssectional survey on the sexuality of 16-20-year-olds in Switzerland. Schweizerische medizinische Wochenschrift 2000;40:1389.

33. Crochard A, Luyts D, di Nicola S and Gonçalves MAG. Self-reported sexual debut and behavior in young adults aged 18-24 years in seven European countries: Implications for HPV vaccination programs. Gynecol.Oncol. 2009;3, Supplement 1:S7-S14.

34. Instituto de la Juventud. Informe Juventud en España 2008 [revista electrónica] [consultado June 29-2010]: Disponible en: http://www.injuve.migualdad.es/injuve/contenidos.item.action?id=1531688780\&menuId=16271008 28.

35. Equipo Daphne. $2^{a}$ Encuesta Shering 2005. Sexualidad y anticoncepción en la juventud española 2005.

36. Layte R, McGee H, Quail A, Rundle K, Cousins G, Donnelly C. et al. The Irish Study of Sexual Health and Relationships. Dublin: Crisis Pregnancy Agency and the Department of Health and Children, 2006.

37. Istituto Superiore di Sanitá. Salute riproduttiva tra gli adolescenti: conoscenze, attitudini e comportamenti 2000.

38. Institute National de la Santé et de la Recherche Medicale, Anrs, INED. Context of sexuality in France. CSF survey 2006.

39. The Northern Ireland Statistics and Research Agency. Health and wellbeing survey 2001.

40. Health Promotion Agency. Towards better sexual health 2002.

41. Finnish Social Science Data Archive. Finnish youth survey 2006.

42. Moreno C, Muñoz-Tinoco V, Pérez P, Sánchez-Queija I, Granado MC, Ramos P. et al. Desarrollo adolescente y salud. Resultados del Estudio HBSC-2006 con chicos y chicas españoles de 11-17 años. Ministerio de Sanidad y Consumo ed. Madrid, 2008.

43. Lavikainen $\mathrm{H}$, Ahlstrom $\mathrm{S}$ and Metso Lea. Relationship between negative experiences and drinking experience among 15-to 16-year-old adolescents in Finland. European addiction research 2008;3:169.

44. Avery L and Lazdane G. What do we know about sexual and reproductive health of adolescents in Europe?. European Journal of Contraception Reproductive Health Care 2008;1:58-70.

45. Weller $\mathrm{S}$ and Davis K. Condom effectiveness in reducing heterosexual HIV transmission. Cochrane Database Syst Rev 2002;1:D003255. 
46. Vaccarella S, Franceschi S, Herrero R, Munoz N, Snijders PJ, Clifford GM. et al. Sexual behavior, condom use, and human papillomavirus: pooled analysis of the IARC human papillomavirus prevalence surveys. Cancer Epidemiol Biomarkers Prev. 2006;2:326-33.

47. de Irala J, Osorio A, Carlos S, Ruiz-Canela M and Lopez-Del Burgo C. Mean Age of First Sex: Do They Know What We Mean?. Arch Sex Behav 2011.

48. Marston $\mathrm{C}$ and King E. Factors that shape young people's sexual behaviour: a systematic review. Lancet 2006;9547:1581-6.

49. Martin T. An European Campaign. The Flying Condom. Martin, Thierry. Plate-forme Prevention SIDA 2006.

50. Ministerio de Sanidad y Política Social. Campañas sobre Salud Sexual [revista electrónica] [consultado 5/31-2010]: Disponible en: http://www.msps.es/ciudadanos/enfLesiones/enfTransmisibles/sida/prevencion/campanas.htm.

51. Halperin DT, Steiner MJ, Cassell MM, Green EC, Hearst N, Kirby D. et al. The time has come for common ground on preventing sexual transmission of HIV. Lancet 2004;9449:1913-5.

52. Green EC. Uganda's HIV Prevention Success: The Role of Sexual Behavior Change and the National Response. AIDS and behavior 2006;4:335.

53. Perales Albert A. ¿Qué menores ven nuestros menores en televisión? 2009.

54. de Irala J, Gómara Urdiain I and López-del Burgo C. Analysis of content about sexuality and human reproduction in school textbooks in Spain. Public Health 2008;10:1093-103.

55. Rivadeneyra $\mathrm{R}$ and Lebo MJ. The association between television-viewing behaviors and adolescent dating role attitudes and behaviors. Journal of adolescence 2008;3:291-305.

56. Brown JD, L'Engle KL, Pardun CJ, Guo G, Kenneavy K and Jackson C. Sexy media matter: Exposure to sexual content in music, movies, television, and magazines predicts black and white adolescents' sexual behavior. Pediatrics 2006;4:1018.

57. L'Engle KL, Brown JD and Kenneavy K. The mass media are an important context for adolescents' sexual behavior. J Adolesc Health 2006;3:186.

58. Skouby SO. Contraceptive use and behavior in the 21 st century: a comprehensive study across five European countries. Eur.J.Contracept.Reprod.Health Care 2010:S42-53.

59. Herlitz C. Sexual risk-taking int he general population of Sweden (1989-2007). Sexual Health 2009:272-80.

60. Centers for Disease Control and Prevention. Condom Fact Sheet in Brief [revista electrónica] [consultado 06/15-2011]: Disponible en: http://www.cdc.gov/condomeffectiveness/brief.html 
